\title{
L'intersexualité chez les mammifères domestiques
}

\author{
EP Cribiu1 1 S Chaffaux² \\ 1 INRA, Laboratoire de Cytogénétique, 78350 Jouy-en-Josas; \\ 2 ENVA, Laboratoire de Pathologie de la Reproduction, 94704 Maisons-Alfort, France
}

(28 Réunion de la Société Française pour l'Étude de la Fertilité; Paris, 19-21 octobre 1989)

\begin{abstract}
Résumé - A l'exception du freo-martinisme bovin, l'intersexualité est rare chez les animaux domestiques. Les quelques cas d'intersexués que nous avons observés chez le chien, les bovins, la chèvre, le mouton et le cheval ont été classés selon leur caryotype. Parmi les intersexués XX sont décrits des chèvres sans corne pseudohermaphrodites mâles et hermaphrodites vrais et des chiens pseudohermaphrodites femelles. Chez les intersexués $X Y$, nous avons étudié un chien hermaphrodite vrai, des chiens pseudohermaphrodites mâles, des juments à dysgénésie gonadique et une vache pseudohermaphrodite femelle. Des intersexués $X X / X Y$ ont été mis en évidence chez des moutons free-martin.
\end{abstract}

\section{cytogénétique / déterminisme du sexe / intersexués / mammiłères domestiques}

Summary - Intersexuality in domestic mammals. With the exception of bovine freemartinism, intersexuality is rarely reported in domestic animals. The few cases of intersexuality reported here in dogs, cattle, goats, sheep and horses were classified according to the karyotype. The XX intersexes described here included goats which were either polled male pseudohermaphrodites or true hermaphrodites and dogs which were female pseudohermaphrodites. Among the XY intersexes studied, one dog was a true hermaphrodite, whereas the others were male pseudohermaphrodites, all mares showed gonadal dysgenesis and one cow was a female pseudohermaphrodite. XX/XY intersexes were detected in ovine cases of freemartinism.

cytogenetics / sexual determinism / intersexes / domestic mammals

\section{INTRODUCTION}

Le sexe génétique des mammifères est fixé au moment de la fécondation et n'est pas influencé par des facteurs de l'environnement. Le mâle est toujours de sexe hétérogamétique $(X Y)$ et la femelle homogamétique $(X X)$. En général, les individus porteurs d'un seul chromosome $X(X O)$ ont un phénotype femelle (Ford et al, 1959; Welshons et Russell, 1959) tandis que la seule présence du gonosome $Y$, quel que soit le nombre de chromosome $X$, entraîne le sexe mâle (Jacobs et
Strong, 1959). L'hypothèse classique est qu'un gène lié au chromosome $Y$, le facteur de détermination testiculaire, TDF, chez l'homme ou TDY chez la souris, induit la formation du testicule. En l'absence du chromosome $Y$, les gènes contrôlant l'ovaire induisent sa formation (Eicher et Washburn, 1986).

En revanche, la différenciation des tractus génitaux interne et externe semble plutôt être contrôlée par les hormones sécrétées par les gonades.

L'intersexualité survient lorsque le déroulement normal de la différenciation des 
gonades, des canaux génitaux et/ou du tractus génital externe est perturbé. Les intersexués sont généralement assimilés à des hermaphrodites possédant des organes génitaux ayant les caractéristiques des deux sexes. Ils sont classés en hermaphrodites vrais, pseudohermaphrodites mâles ou pseudohermaphrodites femelles selon le type du tissu gonadique présent. Les hermaphrodites vrais ayant à la fois du tissu ovarien et testiculaire tandis que les pseudohermaphrodites mâles ont seulement du tissu testiculaire et les pseudohermaphrodites femelles du tissu ovarien.

Dans cet exposé nous décrivons quelques cas d'intersexués que nous avons observés chez les animaux domestiques.

\section{DESCRIPTION DE QUELQUES INTERSEXUÉS OBSERVÉS CHEZ DES ANIMAUX DOMESTIQUES}

\section{Les intersexués à caryotype $\mathrm{XX}$}

Parmi les intersexués à caryotype $X X$, nous avons observé des pseudohermaphrodites mâles et femelles.

\section{Chèvres sans cornes}

Les intersexués $60, X X$, qui sont largement distribués dans les races caprines Saanen et Toggenburg à des fréquences proches de $15 \%$ sont caractérisés par une absence de cornage (Cribiu et Lherm, 1986).

Les anomalies morphologiques varient du mâle presque normal à la femelle presque parfaite. Les premiers ont des testicules scrotaux ou inguinaux avec des dérivés des canaux de Wolff bien développés et des canaux de Müller rudimentaires, en revanche les secondes ont un clitoris hypertrophié, des testicules ou des ovotestis intra-abdominaux, un utérus et un vagin bien développés et de petites vésicules séminales (Corteel et al, 1969). Les chèvres $60, X X$ sans corne que nous avons observées correspondent plutôt à la seconde catégorie.

Des pseudohermaphrodites mâles $X X$ ont également été observés chez le cheval (Cribiu, 1984b), le porc (Hare et Singh, 1979) et le chien (Chaffaux et al, 1980).

\section{Les chiens $X X$}

Parmi les pseudohermaphrodites femelles $X X$ nous avons observé 3 chiennes appartenant à une portée de 5 dont 2 mâles normaux (Chaffaux et Cribiu, 1989).

Les 3 femelles possèdent des appareils génitaux externes présentant une vulve, petite, étroite, dont la muqueuse est blanchâtre et placée en région ventrale. Elle est prolongée vers l'arrière par un repli cutané formant une sorte de fourreau. Le tractus génital interne est celui de femelles normales avec présence sur les ovaires de corps jaune. Le vagin de 5 à $6 \mathrm{~cm}$ de diamètre est très dilaté et rempli d'urine dans les 3 cas observés. Le caryotype de ces 3 animaux est celui d'une femelle normale $78, X X$. Ce sont des pseudohermaphrodites femelles. L'intersexualité a été induite par l'injection de stéroïdes anabolisants à activité androgènique (dynabolon ND : undécanoate de Nandrolone) à la mère pendant la gestation. Ces androgènes ont masculinisé le tractus génital externe en augmentant principalement la distance ano-génitale.

\section{Les intersexués à caryotype $X Y$}

Parmi les syndromes associés au caryotype $X Y$, nous avons observé : un hermaphrodite vrai (Chaffaux et al, 1986), des 
pseudohermaphrodites mâles comprenant des formes classiques (Chaffaux et al, 1980) et une forme particulière à la limite du pseudohermaphrodisme mâle : les dysgénésies gonadiques (Cribiu, 1984a), et enfin un pseudohermaphrodite femelle beaucoup plus rare (Cribiu et al, 1989).

\section{Hermaphrodisme vrai chez un chien $78, X Y$}

Les hermaphrodites vrais qui possèdent à la fois du tissu testiculaire et ovarien sont classés en 3 types : 1) les hermaphrodites latéraux ayant à un testicule d'un côté et un ovaire de l'autre; 2) les hermaphrodites unilatéraux caractérisés par du tissu testiculaire et ovarien (ovotestis) d'un côté, et un ovaire ou un testicule de l'autre; 3) les hermaphrodites bilatéraux définis par l'existence simultané de tissu testiculaire et ovarien (ovotestis) des deux côtés.
La chienne que nous avons observée possède un appareil génital externe composé d'une petite vulve, en position normale, d'un clitoris péniforme très volumineux renfermant un os pénien et enfin d'un vagin très étroit. L'appareil génital interne est composé d'un utérus de taille et de conformation normales, d'un ovaire formé d'un stroma dans lequel se trouvent des follicules atrésiques dépourvus d'ovocytes, et d'une gonade hyperplasiée volumineuse, constituée de tubules séminifères sans cellules germinales, et surmontée d'un épididyme (figs 1,2 ). Cette dernière est porteuse d'une tumeur testiculaire de type sertolinome intratubulaire, observé souvent chez les chiens à testicule ectopique (Chaffaux et al, 1986).

Quelques rares cas d'hermaphrodites vrais de caryotype $X Y$ ont été décrits chez

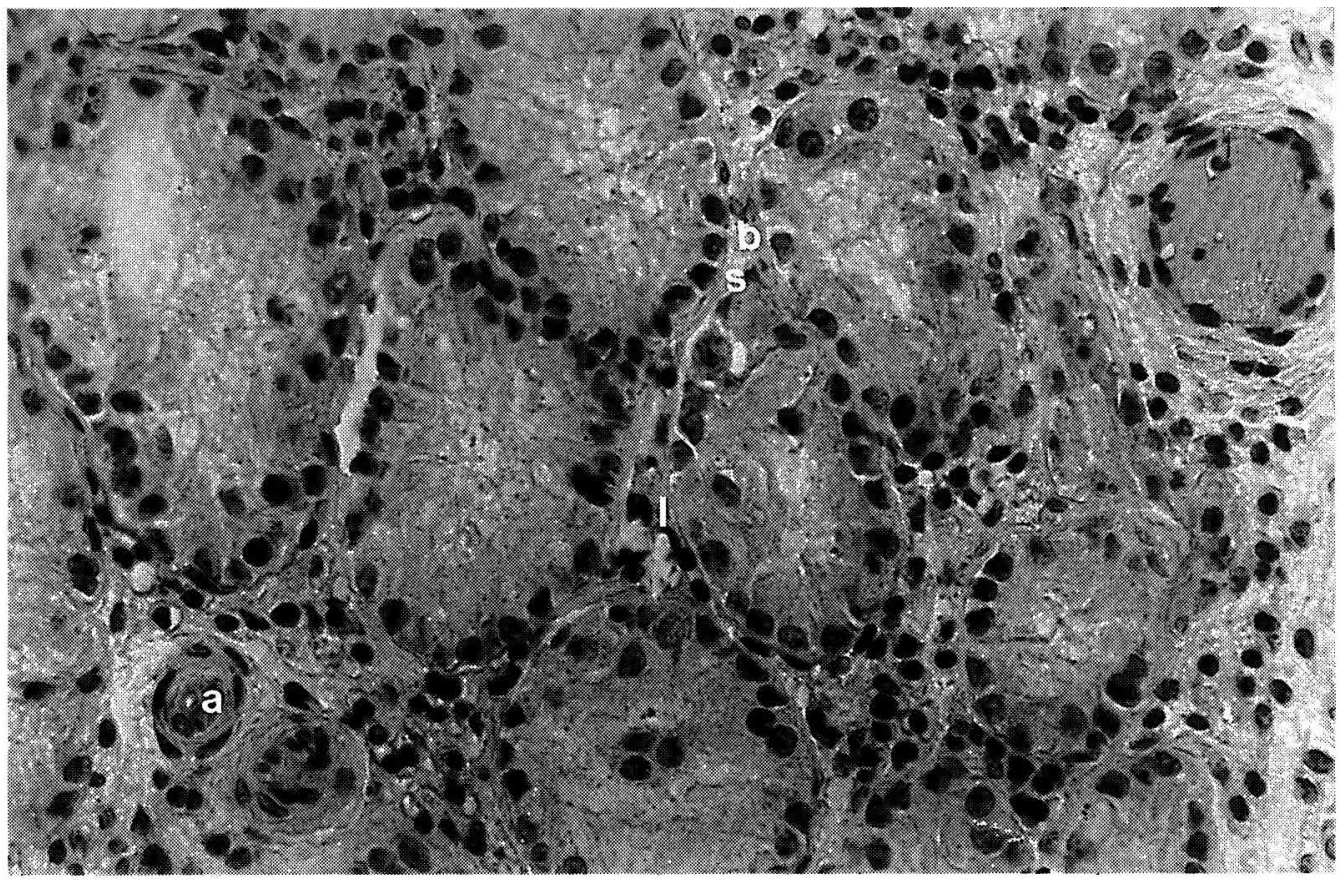

Fig 1. Coupe de testicule d'un chien hermaphrodite ( $x$ 400) (b: basale; s: cellule de Sertoli; : cellule de Leydig; a: artériole). 


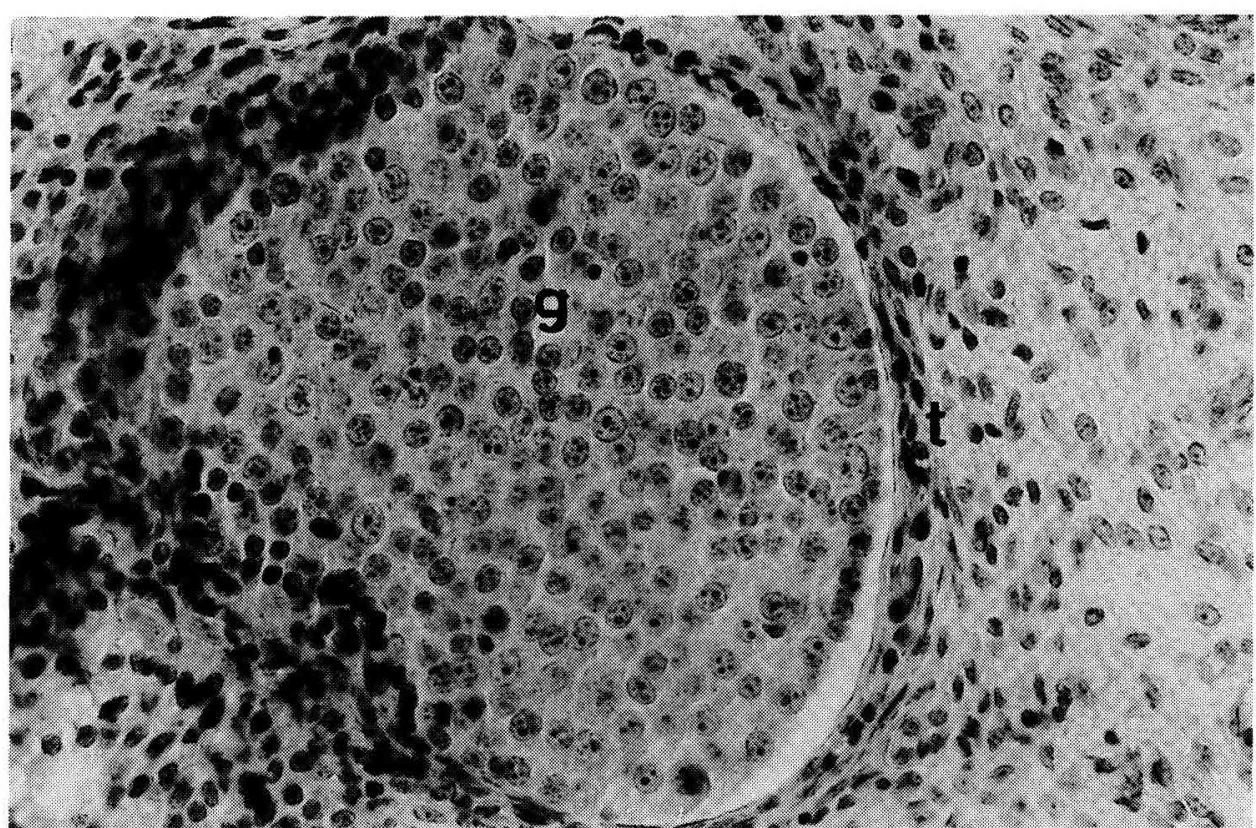

Fig 2. Coupe de l'ovaire d'un chien hermaphrodite $(x 400)$ (g: granulosa, t: thèque).

d'autres espèces domestiques comme les bovins (Switonski et al, 1985).

Les pseudohermaphrodites mâles chez des chiens $78, X Y$

Les gonades des pseudohermaphrodites mâles ne sont composées que de tissu testiculaire.

Nous avons observé 3 cas de pseudohermaphrodisme mâle chez des chiens possédant des testicules en position scrotale, abdominale et inguinale et des formations d'origine müllerienne (utérus) et wolffienne (épididymes). Dans deux cas sur trois la spermatogénèse est arrêtée au stade spermatocytaire et dans un cas elle est complète (Chaffaux et al, 1980). Un cas de pseudohermaphrodisme mâle $X Y$ a été décrit chez le bœuf (Sysa et al, 1974).
Les dysgénésies gonadiques chez les juments $64, X Y$

Parmi les 89 juments subfertiles que nous avons examinées, 2 femelles stériles étaient porteuses d'un caryotype $64, \mathrm{XY}$ (Cribiu, 1984a). Ces 2 juments ont un appareil reproducteur externe femelle anatomiquement normal et un appareil reproducteur interne composé d'un petit col flasque, d'un utérus normal ou légèrement infantile et de petits ovaires. Les cycles œstraux sont très irréguliers ou absents.

De nombreux cas de juments $X Y$ ont été décrits dans la littérature (Cribiu, 1984b). Elles sont de 2 types : les premières, comme les 2 cas que nous avons observés, sont atteintes de dysgénésie gonadique pure et les secondes du syndrome de féminisation testiculaire. Dans 
ce dernier cas, l'appareil reproducteur externe est normal tandis que l'utérus, s'il est présent, est très petit et les gonades intraabdominales sont composées de tissu testiculaire. Ces juments ont quelquefois un comportement et une musculature de mâle et n'ont pas de cycles œstraux.

Des cas de dysgénésie gonadique $X Y$ ont également été décrits chez les bovins (Henricson et Akesson, 1967; Chapman et al, 1978; Sharma et al, 1980; McMillan et al, 1984). Des animaux porteurs de testicule féminisant $X Y$ ont aussi été observés chez les bovins (Hare et Singh, 1979; Long, 1981), les ovins (Cribiu et Matejka, 1985), les caprins (Cribiu et Lherm, 1986) et les porcins (Hare et Singh, 1979).

\section{Les pseudohermaphrodites femelles chez une vache $60, X Y$}

Les pseudohermaphrodites femelles qui ne possèdent que du tissu ovarien sont rarement rencontrés chez les animaux domestiques. Cependant nous avons observé un tel cas chez un animal Charolais né, seul de sa portée, d'une mère fécondée sur œstrus naturel qui n'a subit aucun traitement durant la gestation.

La conformation de l'avant-main, de la tête et l'encolure est plutôt celle d'une femelle, alors que l'arrière-main est de type mâle avec absence de vulve et de fourreau.

L'ensemble de l'appareil génital interne (fig 3) est de type femelle avec un col utérin d'aspect normal, un corps utérin qui se divise rapidement en deux cornes très courtes et des oviductes en continuité avec deux gonades ressemblant à des ovaires de taille et d'aspect normaux pré-

Fig 3. Tractus génital interne de la vache intersexuée (o: ovaire, cu: corps utérin; c: col, v: vagin, mrp: muscle rétracteur du pénis, cp: cavité prépuciale).

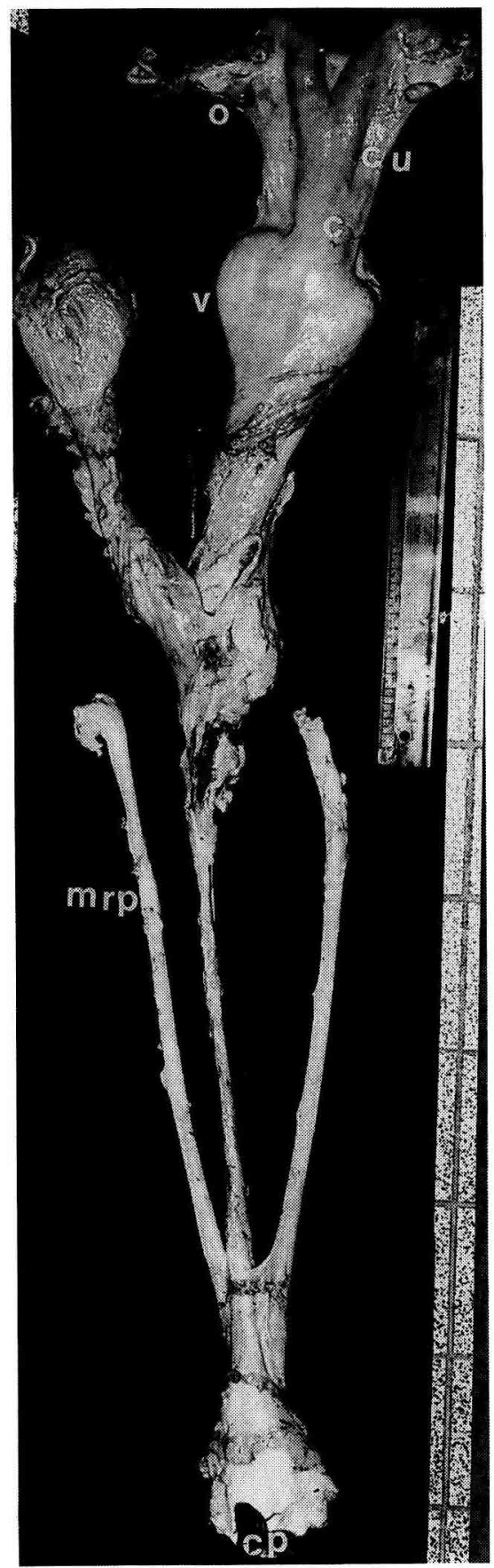


sentant différents follicules dépourvus d'ovocytes. A cet ensemble se trouvent associés des vestiges de l'appareil génital mâle : fourreau, urètre pelvien et muscle rétracteur du pénis. Les profils hormonaux indiquent une absence complète de testostérone, d'œstradiol et de progestérone. Les profils de LH rappelent ceux observés chez des vaches à dysgénésie gonadique (Cribiu et al, 1989).

\section{Les intersexués à caryotype mixoploïde $X X / X Y$}

Les cas de mixoploïdie rencontrés sont de type $X X / X Y$. Selon Hamerton (1971), leur formation résulte de trois mécanismes : 1) un échange de tissu hématopoïétique entre cojumeaux dizygotes hétérosexués, pendant la vie intra-utérine; 2) une fécondation dispermique qui peut être causée par deux mécanismes, d'une part la pénétration d'un spermatozoïde dans l'ovocyte et d'un autre dans le premier globule polaire, et d'autre part la fusion de deux ovules fécondés; 3) d'une succession de non disjonctions mitotiques à partir d'un zygote $X Y$.

\section{Le free-martinisme chez les bovins}

Les intersexués $X X / X Y$ se rencontrent fréquemment chez les bovins en raison du syndrome de free-martinisme dont sont atteintes presque toutes les femelles nées de naissances gémellaires dizygotes hétérosexuées. Son apparition dépend à la fois de la gestation qui doit être gémellaire hétérosexuée et de l'installation d'anastomoses vasculaires placentaires entre les deux fœtus (Marcum, 1974). Si le cojumeau est habituellement normal et fertile, en revanche, la cojumelle, bien que généralement d'aspect externe normal, est stérile et possède un tractus génital interne hypoplasique et/ou plus ou moins masculinisé.

Ainsi dans $98 \%$ des cas des gestations gémellaires dizygotes chez les bovins, des anastomoses consécutives à la fusion allantochoriale s'installent et permettent une circulation fœtale commune qui a pour conséquence l'échange de populations cellulaires génétiquement différentes et d'hormones entre les fœtus. Lorsque les jumeaux sont de sexes différents, on observe deux populations cellulaires leucocytaires $60, X X$ et $60, X Y$ chez le freemartin (femelle stérile) et son cojumeau mâle normal.

\section{Le chimérisme leucocytaire chez les moutons $54, X X / 54, X Y$}

Chez le mouton, les free-martin sont beaucoup plus rares que chez les bovins puisque les anastomoses vasculaires ne s'installent que dans 0,8 à $10 \%$ des cas de gestations gémellaires (Stormont et al, 1953; Alexander et Williams, 1964). L'accroissement du nombre d'agneaux par portée dans certaines races nous a conduit à entreprendre une étude sur les effets du free-martinisme et son incidence dans une race prolifique, la Romanov.

Dans les troupeaux de race Romanov dont la taille de portée est de 2,8 à 3,3 suivant la saison, on observe régulièrement 2 à $3 \%$ d'agnelles infertiles dans les portées mixtes. Nous avons estimé la fréquence du chimérisme $X X / X Y$ et par voie de conséquence du free-martinisme dans un premier lot de 7 brebis stériles et dans un second lot de 118 agnelles issues de portées comprenant au moins un mâle (Matejka et al, 1985, 1987; Chaffaux et al, 1987). Cinq brebis (4 dans le premier lot et 1 dans le second) présentant un chimérisme leucocytaire $X X / X Y$ et un caryotype $X X$ normal dans les tissus non hématopoiétiques laissent apparaître, après abattage, des appa- 
reils génitaux plus ou moins masculinisés (figs 4 et 5). Par conséquent, il semble que le free-martinisme soit un phénomène limité dans cette race puisque sa fréquence peut être estimée à $2 \%(1 / 118)$ et que seulement la moitié des cas de stérilité est causée par sa présence (4/7).

\section{DISCUSSION}

Bien que chez les juments $X O$, un seul chromosome $X$ suffise au bon développement des cordons sexuels corticaux et des structures ovariennes, les deux $X$ sont essentiels pour un développement et un fonctionnement normal des ovaires (Hamerton, 1971).

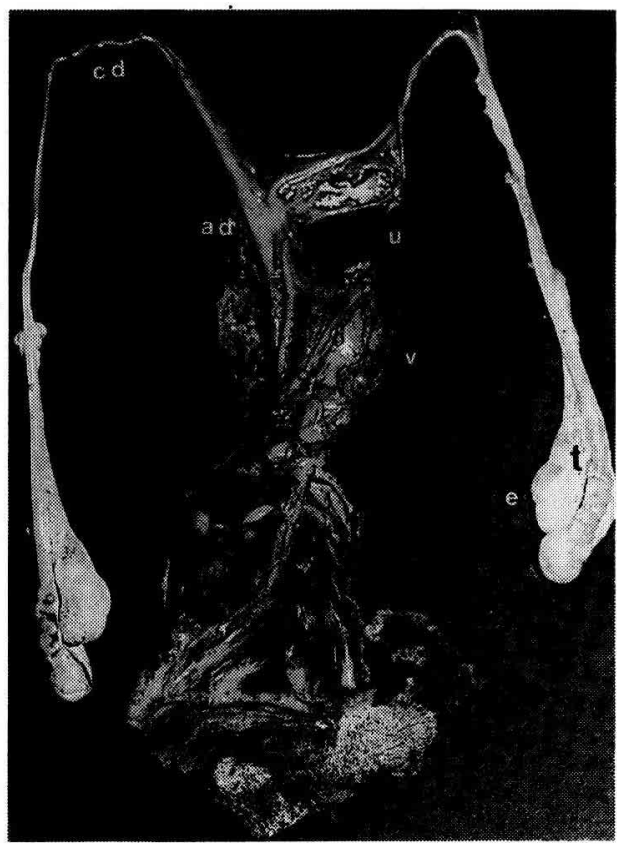

Fig 4. Tractus génital interne d'un mouton freemartin très masculinisé (t: testicule, e: épididyme, cd: canal déférent, ad: ampoule déférente, u: uretère, v: vessie).

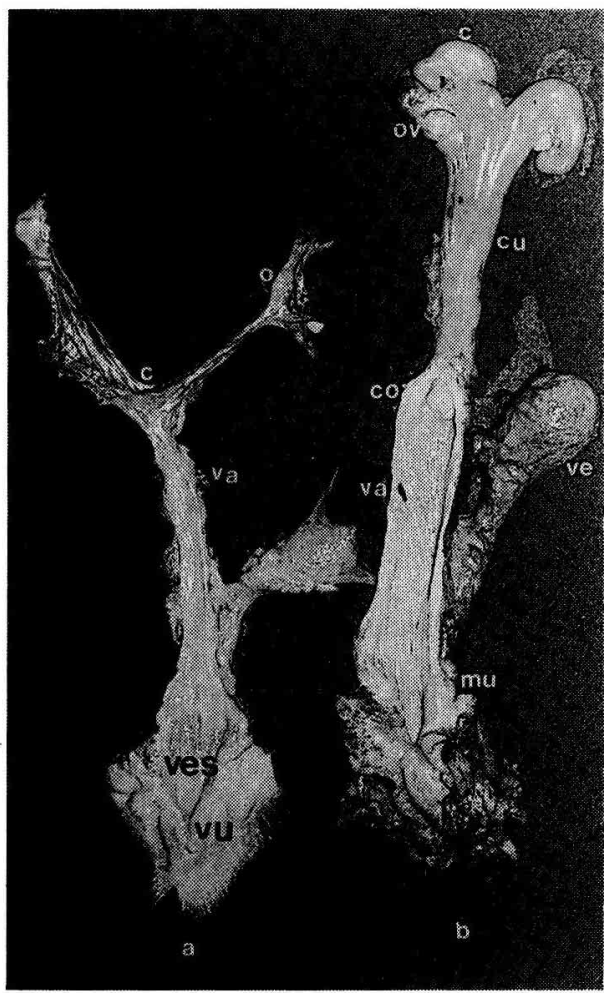

Fig 5. a) Tractus génital interne d'un mouton free-martin peu masculinisé; b) tractus génital interne d'une brebis normale (c: corne, co: col, cu: corps utérin; mu: méat urinaire; o: ovotestis, ov: ovaire, va: vagin, ve: vessie, ves: vestibule, vu: vulve).

Le développement de la gonade indifférenciée en testicule a successivement été attribué à un antigène d'histocompatibilité appelé antigène H-Y (Wachtel et al, 1974, 1975), à des séquences répétitives d'ADN dites Bkm (Epplen et al, 1983; Singh et al, 1984), puis récemment à une protéine en "doigts de zinc" (ZFY) (Page et al, 1987).

L'antigène d'histocompatibilité $\mathrm{H}-\mathrm{Y}$ est commun à plusieurs espèces : I'homme, le rat, le cobaye, le lapin (Wachtel et al, 1974) et le bovin (Ohno et al, 1976). De 
nombreux auteurs ont, tout d'abord, pensé qu'il était codé par un gène situé sur le chromosome $Y$ et qu'il pourrait être responsable (Wachtel et al, 1975) ou très lié au gène responsable du développement testiculaire. Tandis que d'autres le situaient sur un autosome (Kralova et Demant, 1976) ou sur le chromosome $X$ (Bjersing, 1977). II semble à présent que l'antigène $\mathrm{H}-\mathrm{Y}$ ne soit pas impliqué dans la différenciation testiculaire mais qu'il puisse jouer un rôle dans la spermatogénèse après la naissance (Burgoyne et al, 1986).

L'hybridation in situ de sondes contenant des séquences d'ADN du chromosome $Y$ a montré que ces séquences sont souvent présentes dans un des chromosomes $X$ des hommes XX (Guellaen et al, 1984 ) et en revanche absentes dans le chromosome $Y$ de certaines femmes $X Y$ (Disteche et al, 1986). Des travaux récents ont montré que ces séquences contiennent le gène TDF (Page et al, 1987).

Selon Hamerton (1971), l'absence de corne et l'intersexualité des boucs $\mathrm{XX}$ sont transmises par le même allèle ou 2 allèles étroitement liés dont le ou les loci sont situés sur un autosome; la première est transmise comme un caractère simple dominant à pénétrance complète, tandis que la seconde se comporte comme un caractère récessif limité au sexe dont l'expressivité est variable.

De même, la dysgénésie gonadique pure $X Y$ est transmise par les juments à leur descendance; le gène impliqué étant récessif et lié au sexe (Cribiu, 1984a).

La formation du testicule est accompagnée du développement des cellules de Sertoli, des cellules de Leydig et des cellules germinales mâles. L'absence de ces dernières entraîne une stérilité complète chez les intersexués que nous avons étudiés. Cette stérilité pourrait être causée par la dégénérescence des cellules germi- nales due à un déséquilibre dans le dosage de l'X. Dans le testicule, la présence de plus d'un gonosome $X$ dans les cellules germinales entraîne leur dégénérescence. Dans l'ovaire, les deux chromosomes $X$ étant euchromatiques et actifs dans les ovogonies et les ovocytes (Epstein, 1969; Migeon et Jelallian, 1977), la déficience dans le dosage de l' $X$ chez les juments $\mathrm{XY}$, par exemple, pourrait induire un développement folliculaire pauvre qui accélèrerait les processus conduisant à la perte de l'ovocyte (Burgoyne, 1978). Les cellules germinales des fœtus dégénèrent vers la fin de la période foetale et, au moment de la naissance, seul un petit nombre d'ovocytes reste encore dans les ovaires (Carr et al, 1968). Ces ovocytes auront tous disparu à la puberté chez les espèces dont l'intervalle entre leur formation et leur ovulation est long (Lyon et Hawker, 1979; Burgoyne et Bigger, 1976).

Les cellules de Leydig produisent la testostérone qui provoque, d'une part, la différenciation des canaux de Wolff en épididyme, canaux déférents et vésicules séminales et, d'autre part, le développement du tractus génital externe. Les cellules de Sertoli sécrètent l'hormone antimüllérienne $(A M H)$, responsable de la régression des canaux de Müller (Josso et al, 1977) qui pourrait également inhiber le développement des ovaires (Vigier et al, 1987).

En absence d'hormone mâle, un phénotype femelle se développe avec différentiation des canaux de Müller en oviducte, utérus et col utérin et régression des canaux de Wolff (Jost et al, 1973).

La présence des dérivés des canaux de Müller normalement développés chez les animaux à dysgénésie gonadique (XO et $X Y)$ ou chez la vache pseudohermaphrodite femelle implique la non production d'hormone antimüllerienne. En revanche, la présence de tissu testiculaire n'implique 
pas forcément une inhibition des canaux de Müller par l'AMH; celle-ci pouvant ne pas être produite par les cellules de Sertoli.

Les dérivés des canaux de Wolff, étant stimulés par la testostérone sécrétée par les cellules de Leydig n'impliquent pas forcément la présence de testicule, car celleci peut être d'origine exogène, comme dans les cas de pseudohermaphrodisme femelle (testostérone surrénalienne ou tumorale). Enfin, les cas de testicule féminisant (femelle $X Y$ ) ne sont pas causés par un manque de sécrétions testiculaires fœtales, mais par une insensibilité des organes cibles (canaux de Wolff, sinus urogénital et appareil reproducteur externe) à la testostérone (Austin et Short, 1982).

Le syndrome de free-martinisme survient très fréquemment chez les bovins et occasionnellement chez le mouton, le porc et la chèvre. Actuellement, deux hypothèses tentent de l'expliquer. La première, qui est l'héritière de la théorie hormonale fait appel à une substance testiculaire, l'hormone antimüllerienne produite par le cojumeau mâle, qui inhibe à la fois les ovaires et les canaux de Müller; une autre hormone, la testostérone sécrétée d'abord par le cojumeau mâle lors des premiers stades, puis par la gonade du free-martin ensuite, stimulant les canaux de Wolff (Jost et al, 1972; Vigier et al, 1977, 1984). La seconde théorie fait intervenir l'antigène $\mathrm{H}-\mathrm{Y}$, qui serait responsable de la différenciation testiculaire de la gonade. Celui-ci, sécrété par les cellules de Sertoli du testicule du jumeau mâle, passe dans le circulation commune et induit la transformation de la gonade du futur freemartin en testicule (Ohno et al, 1976; Wachtel et al, 1980).

Le développement des techniques cytologiques a permis l'observation détaillée des chromosomes des animaux domestiques et la découverte d'anomalies du ca- ryotype liées à des modifications du phénotype et des troubles de la reproduction. Cependant l'absence d'études cytogénétiques importantes explique sans doute le petit nombre d'anomalies observées car généralement distribuées avec des fréquences faibles; elles exigent pour être détectées, l'établissement du caryotype d'un grand nombre d'animaux.

\section{RÉFÉRENCES}

Alexander G, Williams D (1964) Ovine freemartins. Nature 201, 1296-1298

Austin CR, Short RV (1982) Reproduction in Mammals. Book 2 Embryonic and Fetal Development. Cambridge University Press, New York

Bjersing $L$ (1977) $\mathrm{H}-Y$ antigens and sexual differentiation. Lancet $\mathrm{i}, 311$

Burgoyne PS (1978) The role of sex chromosomes in mammalian germ cell differentiation. Ann Biol Anim Biochim Biophys 18, 317325

Burgoyne PS, Bigger JD (1976) The consequences of dosage deficiency in the germ line: impaired development in vitro of preimplantation embryos from XO mice. Dev Biol 51, 109-117

Burgoyne PS, Levy ER, McLaren A (1986) Spermatogenic failure in male mice lacking $\mathrm{H}-\mathrm{Y}$ antigen. Nature 320, 170-172

Carr DH, Haggar RA, Hart AG (1968) Germ cells in the ovaries of $X O$. female infants. Ann Biol Pathol 49, 521-526

Chaffaux S, Mailhac JM, Cribiu EP, Popescu CP, Cotard JP (1980) L'intersexualité chez le chien (Canis familiaris). A propos de quatre cas. Rec Méd Vét 156, 179-192

Chaffaux S, Cribiu EP, Crespeau F (1986) Un cas rare d'hermaphrodisme vrai latéral chez une chienne 78,XY. Rec Méd Vét 162, 463470

Chaffaux S, Matejka M, Cribiu EP, Crespeau $F$, Ricordeau G (1987) Étude cytogénétique, anatomique et histologique de deux brebis Romanov free-martins. Rec Méd Vét 163, 15-21

Chaffaux S, Cribiu EP (1989) Une famille de trois chiens pseudohermaphrodites femelles 78,XX. Rec Méd Vét (sous presse) 
Chapman HM, Bruère AN, Jaine PM (1978) XY gonadal dysgenesis in a Charolais heifer. Anim Reprod Sci 1, 9-18

Corteel JM, Hulot F, Courot M, Attal J, Philippon A (1969) Examens morphologiques, caryologiques, physiologiques et pathologiques de boucs stériles sans cornes. Ann Génét Sél Anim 1, 341-348

Cribiu EP (1984a) Quelques cas de dysgénésie gonadique chez la jument causés par différentes aberrations gonosomiques (monosomie $X$, mosaïque $X / X X$ et caryotype $X Y$ ). Genet Sel Evol 16, 397-404

Cribiu EP (1984b) Caryotype normal et anomalies chromosomiques du cheval domestique (Equus caballus L). Rec Méd Vét 160, 131139

Cribiu EP, Lherm C (1986) Caryotype normal et anomalies chromosomiques de la chèvre domestique (Capra hircus). Rec Méd Vét 162 , 163-167

Cribiu EP, Matejka M (1985) Caryotype normal et anomalies chromosomiques du mouton domestique (Ovis aries $L$ ). Rec Méd Vét 161, 61-68

Cribiu EP, Chaffaux S, Durand V, Humblot $P$ (1989) Cytogenetic, anatomical, histological and hormonal study of a female pseudohermaphrodite cow. Zuchthygiene 24, 1, 265273

Disteche $\mathrm{CM}$, Casanova $M$, Saal $H$, Friedman C, Sybert V, Graham J, Thuline H, Page DC, Fellous M (1986) Small deletion of the short arm of the $46, X Y$ females. Proc Natl Acad Sci USA 83, 7841-7844

Eicher EM, Washburn LL (1986) Genetic control of primary sex determination in mice. Annu Rev Genet 20, 327-360

Epplen JT, Cellini A, Romero S, Ohno S (1983) An attempt to approach the molecular mechanisms of primary sex determination: $W$ and $Y$-chromosomal conserved simple repetitive DNA sequences and their differential expression in mRNA. J Exp Zool 228, 305312

Epstein CJ (1969) Mammalian oocytes: X chromosomes activity. Science 163, 1078-1079

Ford CE, Miller OJ, Polani PE, De Almeida JC, Briggs JH (1959) A sex-chromosome anomaly in a case of gonadal dysgenesis (Turner's syndrome). Lanceti, 711

Guellaen G, Casanova M, Bishop C, Geldwerth $D$, André $G$, Fellous $M$, Weissenbach $J$ (1984) Human $X X$ males with single copy DNA fragments. Nature $307,172-173$
Hamerton JL (1971) Human Genetics. Academic Press, New York

Hare WCD, Singh EL (1979) Cytogenetics in Animal Reproduction. Commonwealth Agricultural Bureaux

Henricson B, Akesson A (1967) Two heifers with gonadal dysgenesis and the sex chromosomal constitution XY. Acta Vet Scand 8, 262272

Jacobs PA, Strong JA (1959) A case of human intersexuality having a possible $X X Y$ sexdetermining mechanism. Nature 183, 302303

Josso N, Picard JY, Tran D (1977) The antimüllerian hormone. Rec Prog Horm Res 33, 117 167

Jost A, Vigier B, Prepin J (1972) Freemartins in cattle: the first steps of sexual organogenesis. J Reprod Fertil 29, 349-379

Jost A, Vigier B, Prepin J, Perchellet JP (1973) Studies on sex differentiation in mammals. Rec Prog Horm Res 29, 1-35

Kralova J, Demant P (1976) Expression of $\mathrm{H}-\mathrm{Y}$ antigen on thymus cells and skin: differential genetic control linked to $\mathrm{K}$ end of $\mathrm{H}-2$. I $\mathrm{mmu}$ nogenetics 3, 583-594

Long SE (1981) Testicular feminisation in an Ayrshire cow. Vet Rec 109, 116-118

Lyon MF, Hawker SG (1979) Reproductive lifespan in irradiated and unirradiated chromosomally XO mice. Genet Res 21, 185-194

Marcum JB (1974) The freemartin syndrome. Anim Breeding Abstr 42, 227-242

Matejka M, Cribiu EP, Chaffaux S, Schmitt O, Ricordeau G (1985) Deux cas de freemartinisme chez des moutons Romanov (Ovis aries). Genet Sel Evol 17, 419-434

Matejka M, Cribiu EP, Ricordeau G, Chaffaux S (1987) Fréquence du free-martinisme chez les agnelles Romanov. Rec Méd Vêt 163, 635-638

McMillan KL, Fielden ED, McNatty KP, Henderson HV (1984) LH concentrations in two cattle with $X Y$ gonadal dysgenesis. J Reprod Fertil 71, 525-531

Migeon BR, Jelallian K (1977) Evidence for two active $X$ chromosomes in germ cells of female before meiotic entry. Nature 269, 242243

Ohno S, Christian LC, Wachtel SS, Koo GC (1976) Hormonal-like role of $\mathrm{H}-\mathrm{Y}$ antigen in bovine freemartin gonad. Nature 261, 597 599

Page DC, Mosher R, Simpson EM, Fisher EMC, Mardon G, Pollack J, McGillvray B, de la 
Chapelle A, Brown LG (1987) The sexdetermining region of the human $Y$ chromosome encodes a finger protein. Cell 51, 1091-1104

Sharma AK, Vijaykumar NK, Khar SK, Verma SK, Nigam JM (1980) XY gonadal dysgenesis in a heifer. Vet Rec 107, 328-330

Singh L, Phillips C, Jones KW (1984) The conserved nucleotide sequences of $B \mathrm{~km}$, which define Sxr in the mouse, are transcribed. Cell 36, 111-120

Stormont C, Weir WC, Lane LL (1953) Erythrocyte mosaicism in pair of sheep twins. Science 118, 695-696

Switonski M, Czaplicki R, Houszka M (1985) A case of an intersexuality in cattle. Med Vet 41, 302-304

Sysa P, Bernacki Z, Kunska A (1974) Intersexuality in cattle. A case of male pseudohermaphroditismus with a $60, X Y$ karyotype. Vet $\operatorname{Rec}$ 94, 30-31

Vigier B, Prepin J, Perchellet JP, Jost A (1977) Le développement de l'effet free-martin chez le fœetus de veau. Ann Méd Vét 121, 521-536
Vigier B, Tran D, Legeai L, Bezard J, Josso N (1984) Origin of anti-Müllerian hormone in bovine freemartin fetuses. J Reprod Fertil 70 , 473-479

Vigier B, Warin F, Magre S, Tran D, Josso N (1987) Purified bovine AMH indices a characteristic freemartin effect in fetal rat prospective ovaries exposed to it in vitro. Development 100, 43-55

Wachtel SS, Koo GC, Zuckerman EE, Hammerling U, Scheid MP, Boyse EA (1974) Serological cross-reactivity between $\mathrm{H}-\mathrm{Y}$ (male) antigens of mouse and man. Proc Natl Acad Sci USA 71, 1215-1218

Wachtel SS, Ohno S, Koo GC, Boyse EA (1975) Possible role for $\mathrm{H}-\mathrm{Y}$ antigen in the primary determination of sex. Nature 257, 235-236

Wachtel SS, Hall JL, Muller U, Chaganti RSK (1980) Serum-borne $H-Y$ antigen in the fetal bovine freemartin. Cell 21, 917-926

Welshons WJ, Russell LB (1959) The Y chromosome as the bearer of male determining factors in the mouse. Proc Natl Acad Sci USA $45,560-566$ 